\title{
Transmission of Lamb waves across a partially closed crack: Numerical analysis and experiment
}

\author{
AUTHOR(S):
}

Matsushita, Mutsuki; Mori, Naoki; Biwa, Shiro

\section{CITATION:}

Matsushita, Mutsuki ...[et al]. Transmission of Lamb waves across a partially closed crack: Numerical analysis and experiment. Ultrasonics 2019, 92: 57-67

\section{ISSUE DATE:}

2019-02

URL:

http://hdl.handle.net/2433/236057

\section{RIGHT:}

(c) $<2019>$ This manuscript version is made available under the CC-BY-NC-ND 4.0 license

http://creativecommons.org/licenses/by-nc-nd/4.0/:; The full-text file will be made open to the public on 01 February 2021 in accordance with publisher's 'Terms and Conditions for Self-Archiving'.; This is not the published version. Please cite only the published version.; この論文は出版社版でありません。引用の際には出版社版をご磪認じ利用ください。 
This manuscript has been published in Ultrasonics, Vol. 92 (February 2019), pp. 57-67.

DOI: $10.1016 /$ j.ultras.2018.09.007

Transmission of Lamb waves across a partially closed crack: Numerical analysis and experiment Mutsuki Matsushita, ${ }^{a}$ Naoki Mori, ${ }^{\text {a, b }}$ Shiro Biwa ${ }^{a, *}$

a Department of Aeronautics and Astronautics, Graduate School of Engineering, Kyoto University, Katsura, Nishikyo-ku, Kyoto 615-8540, Japan.

${ }^{\mathrm{b}}$ Present address: Department of Mechanical Engineering, College of Science and Engineering, Ritsumeikan University, Kusatsu 525-8577, Japan.

* Corresponding author. Tel: +81-75-383-3796.

E-mail address: biwa@kuaero.kyoto-u.ac.jp (S. Biwa).

\begin{abstract}
The transmission characteristics of Lamb waves across a partially closed through-thickness crack in a plate are investigated numerically and experimentally. In the numerical analysis, the spectral element method is used to simulate the transmission of the lowest-order symmetric (S0) and antisymmetric (A0) Lamb modes across a crack in a low-frequency range. The analysis is carried out for an open crack with traction-free surfaces as well as for a partially closed crack modeled as a spring-type interface characterized by normal and tangential stiffnesses. The transmission ratios of both modes are obtained from the spectral amplitude of the simulated transmission waveforms for different crack lengths and interfacial stiffnesses. The numerical results show that the transmission ratio of the S0 mode increases monotonically with the interfacial stiffness, but that of the A0 mode depends on the interfacial stiffness in a non-monotonic manner depending on the frequency. The Lamb wave transmission measurements are carried out for aluminum alloy plates with artificial slits or a fatigue crack. The experimental results for the plates with slits show reasonable agreement with the numerical results for open cracks. The measured transmission ratio of the $\mathrm{S} 0$ mode is shown to decrease with the tensile load applied to the plate, but that of the A0 mode shows different load dependence for different frequencies. The qualitative features of the experimental results for the fatigue crack are discussed based on the numerical simulation for closed cracks.
\end{abstract}

Key words: Structural health monitoring; Lamb wave; Closed crack; Spectral element method; Spring-type interface model 


\section{Introduction}

Elastic guided waves propagate on thin-walled structures with relatively low loss of energy and offer an effective means for detecting and characterizing defects in such structures. Lamb waves are typical guided wave modes which can be used for nondestructive evaluation and structural health monitoring of thin plate structures. Their multi-modal and dispersive characteristics, which need to be properly taken into account in the evaluation process, are well documented in the existing literature $[1,2]$. Complicated scattering phenomena of Lamb waves at various types of defects have been studied by many investigators [3-8]. Furthermore, different defect detection and characterization algorithms using Lamb waves have also been proposed $[9,10]$.

For structural components used in cyclic loading environments such as airframes, the Lamb wave-based monitoring of fatigue cracks has been an issue of practical importance [11-17]. Among others, Ihn and Chang $[12,13]$ investigated the use of a built-in sensor/actuator network to monitor the fatigue crack growth at riveted joints and repair patches. Cho and Lissenden [17] examined the Lamb wave transmission across a fatigue crack growing from a fastener hole for different transmitter-receiver paths. In actual structures, fatigue cracks are often closed with their surfaces in partial contact with each other, which influences the acquired signals of Lamb waves [16]. In the above-mentioned work [17] it was also noted that the Lamb wave transmission behavior across a fatigue crack was different when tensile loading was applied to the plate, indicating that the crack was partially closed. The influence of partial closure of fatigue cracks on the ultrasonic wave scattering characteristics has been a long-standing issue in ultrasonic nondestructive testing [18-20]. In order to further advance the Lamb wave-based monitoring of fatigue cracks, it is important to fully understand the effect of crack closure on the interaction of Lamb waves with them. While the contact acoustic nonlinearity of Lamb waves at fatigue cracks is attracting current attention [21, 22], even the fundamental linear phenomena related to the Lamb wave interaction with closed cracks remain largely unexplained.

Recently, Mori and Biwa [23] studied the transmission characteristics of the lowest-order symmetric (S0) and antisymmetric (A0) Lamb waves at the contacting edges of two plates experimentally. In their experiments, the transmission coefficient of the S0 mode showed monotonic dependence on the applied pressure and the wave frequency, but the transmission coefficient of the A0 mode exhibited non-monotonic behavior showing local minima at a certain pressure and frequency. Furthermore, the features of the experimental results were explained qualitatively by the theoretical analysis in which the contacting interface was modeled as distributed springs in the normal and tangential directions (spring-type interface model) [24-26]. This work provides some useful insights into the Lamb wave interaction with a partially closed crack, since the contacting edges of plates represent the local state of a 
through-thickness crack of the plate which is closed under in-plane compressive stresses. It then appears intriguing to examine the Lamb wave transmission across a partially closed crack in a plate from a theoretical point of view based on the spring-type interface model.

In this study, the transmission characteristics of the lowest-order Lamb modes across an open crack as well as a partially closed crack are investigated by numerical simulations. The partially closed crack is modeled as a spring-type interface, and its interfacial stiffnesses are varied in order to examine the effect of crack closure on the Lamb wave transmission. The Lamb wave transmission measurements are carried out for artificial slits and for a fatigue crack in aluminum alloy plates. Different tensile loads are applied to the plate to examine the effect of the crack closure on the transmission characteristics of Lamb waves. This paper is structured as follows. In Section 2, the numerical model of the Lamb wave propagation in an elastic plate with a through-thickness crack is presented, and the numerical simulation procedure using the spectral element method [27-30] is outlined. The corresponding Lamb wave transmission measurements are described in Section 3. In Section 4, the numerical and experimental results for open cracks (artificial slits), the numerical results for closed cracks and the experimental results for the fatigue crack are presented. Discussions of the numerical and experimental results are given in Section 5, and concluding remarks in Section 6.

\section{Numerical analysis}

\subsection{Modeling of Lamb wave propagation}

The schematic of the numerical model considered in this study is shown in Fig. 1. A thin flat plate (thickness $H$, length $L$ and width $W$ ) made of a homogeneous and isotropic linear elastic solid (mass density $\rho$, longitudinal and shear wave velocities $c_{\mathrm{L}}$ and $c_{\mathrm{T}}$, respectively) occupies the region $-L / 2<x<$ $L / 2,0<y<W$ and $0<z<H$ in the Cartesian $x-y-z$ coordinate system. The wave motion in the plate is analyzed based on the linear theory of elastodynamics assuming infinitesimal strains and Hooke's law. The plate has a straight, through-thickness crack of length $C$ at $x=0$ extending parallel to the $y$-axis, on which the displacements can be discontinuous. The crack is assumed to be either open or partially closed. The open crack is assumed to have traction-free surfaces, i.e.,

$$
\begin{aligned}
& \sigma_{x}\left(0^{+}, y, z, t\right)=\sigma_{x}\left(0^{-}, y, z, t\right)=0, \\
& \tau_{x y}\left(0^{+}, y, z, t\right)=\tau_{x y}\left(0^{-}, y, z, t\right)=0, \\
& \tau_{x z}\left(0^{+}, y, z, t\right)=\tau_{x z}\left(0^{-}, y, z, t\right)=0,
\end{aligned}
$$


where $\sigma_{x}(x, y, z, t), \tau_{x y}(x, y, z, t)$ and $\tau_{x z}(x, y, z, t)$ are the stress components and $t$ denotes the time. The partially closed crack is modeled as a spring-type interface with normal and tangential stiffnesses $K_{\mathrm{N}}$ and $K_{\mathrm{T}}$, respectively $[24,25]$, i.e.,

$$
\begin{aligned}
& \sigma_{x}\left(0^{+}, y, z, t\right)=\sigma_{x}\left(0^{-}, y, z, t\right)=K_{\mathrm{N}}\left[u_{x}\left(0^{+}, y, z, t\right)-u_{x}^{-}\left(0^{-}, y, z, t\right)\right] \\
& \tau_{x y}\left(0^{+}, y, z, t\right)=\tau_{x y}\left(0^{-}, y, z, t\right)=K_{\mathrm{T}}\left[u_{y}\left(0^{+}, y, z, t\right)-u_{y}\left(0^{-}, y, z, t\right)\right] \\
& \tau_{x z}\left(0^{+}, y, z, t\right)=\tau_{x z}\left(0^{-}, y, z, t\right)=K_{\mathrm{T}}\left[u_{z}\left(0^{+}, y, z, t\right)-u_{z}\left(0^{-}, y, z, t\right)\right],
\end{aligned}
$$

where $u_{x}(x, y, z, t), u_{y}(x, y, z, t)$, and $u_{z}(x, y, z, t)$ are the displacement components. The interfacial stiffnesses $K_{\mathrm{N}}$ and $K_{\mathrm{T}}$ represent the extent of crack closure. The conditions of Eq. (2) include those of an open crack in Eq. (1) as a special case when $K_{\mathrm{N}}=K_{\mathrm{T}}=0$. Later in Section 6, the case where the crack has both open and closed parts will be discussed in comparison to the experimental results.

In recent works on structural health monitoring using Lamb waves, surface-mounted or built-in piezoelectric transducers are mainly used. In the present study, however, the wave excitation and detection using the oblique incidence/transmission through wedges are dealt with in order to examine the transmission of the two lowest-order Lamb modes ( $\mathrm{S} 0$ and $\mathrm{A} 0$ ) across the crack separately. The wedge-based Lamb wave excitation/detection is simulated in the numerical analysis in the following simplified manner. As the excitation signal, the out-of-plane nodal force $g\left(t-t_{\mathrm{d}}(x)\right)$ is prescribed with a position-dependent time delay $t_{\mathrm{d}}$ on the rectangular area denoted by $\mathrm{P},|x+L / 4|<L_{x} / 2,|y-W / 2|<L_{y} / 2$ on the upper surface $z=H$, where $L_{x}$ and $L_{y}$ denote the dimensions of the excitation area. The function $g(t)$ denotes the excitation waveform and the delay is given by $t_{\mathrm{d}}(x)=(x+L / 4) \sin \theta / c_{\mathrm{w}}$ according to the $x$-coordinate of each node in the area $\mathrm{P}$, where $\theta$ is the simulated incident angle and $c_{\mathrm{w}}$ is the velocity of the longitudinal wave in the wedge. For simplicity, this out-of-plane force is assumed to have a constant magnitude irrespective of the node position in the area $\mathrm{P}$, and its magnitude is chosen arbitrarily due to the linear nature of the problem. The exterior surfaces of the plate are assumed traction-free except the excitation area $\mathrm{P}$. The time-dependent excitation force on the area $\mathrm{P}$ generates dynamic motion in the plate, which propagates as Lamb waves. The rectangular area $\mathrm{Q}\left(|x-L / 4|<L_{x} / 2,|y-W / 2|<L_{y} / 2\right.$ on the upper surface) is regarded as the detection area, and the transmission waveform is obtained as the integral of the out-of-plane nodal displacement over the area $\mathrm{Q}$ with the similar position-dependent delay.

\subsection{Numerical implementation}

The numerical simulations are carried out for an aluminum alloy plate $\left(\rho=2700 \mathrm{~kg} / \mathrm{m}^{3}, c_{\mathrm{L}}=6.2 \mathrm{~km} / \mathrm{s}\right.$, $c_{\mathrm{T}}=3.1 \mathrm{~km} / \mathrm{s}$ ) with the dimensions $L=300 \mathrm{~mm}, W=80 \mathrm{~mm}$, and $H=5 \mathrm{~mm}$ according to the experiments described below. The three-dimensional analysis of the elastic wave propagation in the plate is performed 
using the spectral element method proposed originally by Patera [27]. Detailed formulations of this method and applications to the problems of elastic wave propagation in plates are described in Refs. [28-30]. An advantage of this method is that the mass matrix becomes diagonal [28-30], which is beneficial for the computational efficiency.

On the crack surfaces $(x=0,0<y<C)$, double nodes are arranged which have separate nodal displacement components, and the boundary conditions of Eq. (1) or (2) are enforced on each node pair on the crack surfaces. The numerical analysis is carried out for different values of crack length $C$ in order to examine its effects on the Lamb wave transmission. In the present analysis, the interfacial normal and tangential stiffnesses are assumed constant irrespective of the position on the crack surfaces. Foregoing studies on the ultrasonic wave reflection/transmission at solid-solid contacting interfaces [25, 31-34] have revealed that the ratio of two interfacial stiffnesses $K_{\mathrm{T}} / K_{\mathrm{N}}$ can take a range of values between 0.2 and 0.6 . In the present analysis, however, the ratio is fixed as one of typical values $K_{\mathrm{T}} / K_{\mathrm{N}}=0.3$ in order to represent the magnitude of interfacial stiffnesses in terms of a single parameter $K_{\mathrm{N}}$.

A burst signal of the frequency $0.25 \mathrm{MHz}$ with the amplitude modulated by a Gaussian function is used as the excitation waveform $g(t)$. The dispersion curves for the $5 \mathrm{~mm}$-thick aluminum alloy plate are shown in Fig. 2. At the frequency of $0.25 \mathrm{MHz}$, the A0 mode has the shortest wavelength $(9.88 \mathrm{~mm})$ among the propagating guided wave modes ( $\mathrm{S} 0$ and $\mathrm{A} 0 \mathrm{Lamb}$ modes and the lowest-order shear horizontal mode SH0). In the present analysis, the dimensions of all elements are set as $5 \mathrm{~mm}$ ( $x$-direction) $\times 5 \mathrm{~mm}$ $(y$-direction $) \times 1.25 \mathrm{~mm}(z$-direction $)$, and the numbers of GLL points in each element are chosen as $N_{\xi}=6$, $N_{\eta}=6$ and $N_{\zeta}=3$. In this case the average distance between two neighboring nodes in the in-plane direction is around one-tenth of the wavelength of the A0 mode at $0.25 \mathrm{MHz}$. The total number of elements is 3,840 and the total number of nodes is 220,104 at maximum (for the longest crack). The time integration is carried out using the fourth-order Runge-Kutta method with the time increment $10 \mathrm{~ns}$ to satisfy the stability condition [30].

The piezoelectric transducers used in the experiments have the length $18 \mathrm{~mm}$ and width $16 \mathrm{~mm}$. The dimensions of the excitation and detection areas are then given as $L_{x}=L_{\mathrm{tr}} / \cos \theta$, where $L_{\mathrm{tr}}=18 \mathrm{~mm}$, and $L_{y}$ $=16 \mathrm{~mm}$ simply assuming a parallel beam in the wedge. Two different incident angles are considered in the numerical analysis in order to simulate the excitation/detection of the S0 and A0 Lamb modes. The velocities of the $\mathrm{S} 0$ and $\mathrm{A} 0$ modes at $0.25 \mathrm{MHz}$ are shown in Table 1, and the longitudinal wave velocity in the PMMA wedge is $c_{\mathrm{w}}=2.7 \mathrm{~km} / \mathrm{s}$. For the analysis of the $\mathrm{S} 0$ mode excitation, Snell's law gives the incident angle $\theta=31 \mathrm{deg}$ which gives $L_{x}=21 \mathrm{~mm}$. For the A0 mode excitation, there is no incident angle satisfying Snell's law, so the angle $\theta$ is set as $75 \mathrm{deg}$ which was the angle used in the experiment. In this 
case, the value of $L_{\mathrm{tr}} / \cos \theta$ exceeds the dimension of the base plane of the wedge in the $x$-direction (28 $\mathrm{mm}$ ), so $L_{x}$ is replaced by the latter, $L_{x}=28 \mathrm{~mm}$.

For the cases of $\theta=31 \mathrm{deg}$ (S0 mode incidence) and $\theta=75 \mathrm{deg}$ (A0 mode incidence), the calculated distributions of the out-of-plane displacement on the upper surface of the plate are shown in Fig. 3 at certain elapsed times when the main wave packet of the S0 or A0 mode has not yet arrived at the crack located at $x=0$. Note that only the left half $(x<0)$ of the whole model is displayed. In Fig. 3, the transverse profiles of these distributions at $x=-30 \mathrm{~mm}$ are also shown. It is noted that the incident wave field in each case is not uniform in the $y$-direction. It is also observed that the wave packet of A0 mode has a narrower main lobe than that of S0 mode, due to the shorter wavelength of the former mode. In the present study, the Lamb wave-crack interaction is analyzed for these fixed incident wave fields. As a consequence, the findings obtained in the present analysis are applicable to the particular excitation/detection conditions and the relative location of the crack for the given plate geometry.

In order to confirm that the spring-type interface model is properly incorporated in the numerical analysis, the results for the case where the partially closed crack is extended over the entire width of the plate $(C / W=1)$ were compared to those of the two-dimensional analysis for the spring-type interface joint of plates [26]. For this purpose, the out-of-plane displacement waveform was calculated at $x=75 \mathrm{~mm}$ on the center line $(y=40 \mathrm{~mm}$ ) of the upper surface for each of the S0 and A0 mode incidence (note that the waveform was computed at this single point, not as the integral over the detection area $\mathrm{Q}$ as mentioned above which is regarded as the transmission waveform in the subsequent discussions). The wave packet of the S0 (A0) mode was selected from the transmitted waveform for each mode by an appropriate window, and its amplitude spectrum was computed by the fast Fourier transform (FFT). The spectral amplitude at $0.25 \mathrm{MHz}$ was normalized by the corresponding spectral amplitude obtained for the homogeneous plate without a crack and considered as the transmission coefficient of each mode. The so-obtained transmission coefficients of the S0 and A0 modes for different interfacial stiffnesses are shown in Fig. 4 together with the transmission coefficients obtained by the two-dimensional analysis using the hybrid finite element method [26]. It can be observed that the results by the present numerical scheme are in reasonable agreement with the two-dimensional analysis, demonstrating that the spring-type interface condition is properly implemented in the numerical analysis.

\section{Experiment}

\subsection{Specimens}

The Lamb wave transmission measurements were carried out as in the numerical simulations demonstrated above. The schematic of the plate specimens is shown in Fig. 5. The plates were made of 
A5052 aluminum alloy and their dimensions were the same as the numerical model. Three types of specimens were used in the experiment: (i) a plate with no defect employed as the reference plate, (ii) plates with an artificial slit of the lengths 10,20,30,40,50, 60 and $70 \mathrm{~mm}$ made by electric discharge machining (EDM) as shown in Fig. 5(a), and (iii) a plate with a $46 \mathrm{~mm}$-long fatigue crack grown from a $10 \mathrm{~mm}$-long EDM slit as shown in Fig. 5(b). The EDM slits were made in the plate width direction, and had the gap opening of about $0.5 \mathrm{~mm}$. The fatigue crack was grown from the EDM slit by cyclic three-point bending with the bending moment about the $z$-axis, and its inclination was within 2 degree from the $y$-axis (three plate specimens were prepared and subjected to cyclic bending to introduce a fatigue crack, and the one with the straightest crack was chosen for the experiment). Since the plate with the fatigue crack had the total defect length of $56 \mathrm{~mm}$ (the EDM slit $10 \mathrm{~mm}$ and the fatigue crack $46 \mathrm{~mm}$ ), the specimen with a $56 \mathrm{~mm}$-long EDM slit was also prepared to examine the difference between the EDM slit and the fatigue crack.

\subsection{Lamb wave measurement}

The schematic of the Lamb wave measurement is shown in Fig. 6. The same Gaussian pulse of the center frequency $0.25 \mathrm{MHz}$ as in the numerical simulations was sent from an Agilent arbitrary waveform generator 33220A to the emitting transducer via a Thamway amplifier T145-7516B and a RITEC attenuator RA-30. Piezoelectric longitudinal wave transducers (KGK, nominal frequency $0.25 \mathrm{MHz}$ ) were fitted to adjustable-angle PMMA wedges (length $28 \mathrm{~mm}$ and width $30 \mathrm{~mm}$ ) and mounted on the plate surface with coupling medium (glycerin) to emit and to receive the ultrasonic wave. In the same manner as in the numerical simulations, the angles of the wedges were set as $31 \mathrm{deg}$ and $75 \mathrm{deg}$, respectively, to excite and detect the S0 or A0 Lamb mode dominantly. The transmitted wave detected with the receiving transducer was saved in a personal computer after averaging over 64 synchronized signals in a Keysight oscilloscope DSOS054A.

The measurements for the plates with the EDM slits of the lengths 10 to $70 \mathrm{~mm}$ were carried out by placing the experimental setup on a flat table. On the other hand, the measurements for the plate with the fatigue crack and the plate with the $56 \mathrm{~mm}$-long slit were performed by applying tensile load in the direction normal to the crack (slit), in order to examine its effect on the Lamb wave transmission characteristics. An Instron-type tensile testing machine (Shimadzu Autograph AG-Xplus) was used to apply the tensile load to the plate. The plate was held vertically and clamped at both ends by the chucks of the testing machine. The chucked areas on the plate are shown in Figs. 5 and 6. The wedges were fixed on the plate using plastic tie bands. The tensile force was measured by a load cell equipped in the testing machine. When the plate was first hung by the upper chuck, the value of the load cell was reset to zero. 
When both ends of the plate were clamped, the load cell indicated a non-zero force $(50 \mathrm{~N}$ in the S0-mode measurement and $54 \mathrm{~N}$ in the $\mathrm{A} 0$-mode measurement): this was the initial load from which the load was varied. The tensile load was increased from the initial value up to $400 \mathrm{~N}$ at the crosshead speed of $1 \mathrm{~N} / \mathrm{s}$, and then decreased down to $0 \mathrm{~N}$ at the same crosshead speed. During the loading/unloading process, the Lamb wave measurement was carried out at every $10 \mathrm{~N}$ by holding the tensile load constant for 20 seconds.

\section{Numerical and experimental results}

4.1 Plate with an open crack

The transmission behavior of the S0 and A0 modes in the plate with an open crack is first examined. For the excitation of the $\mathrm{S} 0$ and $\mathrm{A} 0$ modes at the center frequency of $0.25 \mathrm{MHz}$, the numerically simulated transmission waveforms for three different crack lengths are shown in Fig. 7 (a) and (b), respectively, together with the results for a homogeneous reference plate without a crack $(C=0)$. The crack length $C$ is normalized by the plate width $W$. According to the group velocities shown in Table 1, the main wave packets in the reference plate at $43 \mu \mathrm{s}$ in Fig. 7(a) and at $58 \mu \mathrm{s}$ in Fig. 7(b) correspond to the S0 and A0 modes, respectively. It is shown that the magnitudes of both modes decrease as the length of the crack increases.

The measured voltage waveforms for the reference plate and the plates with the EDM slit of length 20, 40, and $60 \mathrm{~mm}$ are shown in Fig. 7(c) and (d). The main wave packets for the reference plate located at around $114 \mu \mathrm{s}$ in Fig. 7(c) and at around $127 \mu \mathrm{s}$ in Fig. 7(d) correspond to the S0 and A0 modes, respectively. The arrival times of these modes in the measured waveforms are different from those in the numerical results as the former include the propagation time within the wedges. It can be seen that the peak voltages of the measured waveforms decrease as the length of the slit increases in qualitative agreement with the numerical results in Fig. 7(a) and (b), which is expected since the open crack (slit) will hinder the wave transmission.

From the transmission waveforms, the amplitude spectra of the S0 and A0 mode signals are calculated by the FFT using a Gaussian window. The transmission ratio of each mode is defined as the spectral amplitude $A(f)$ for the plate with a crack divided by $A_{0}(f)$ for the reference plate as

$$
T(f)=\frac{A(f)}{A_{0}(f)}
$$

where $f$ denotes the frequency. For the numerical simulation and the experiment, the transmission ratios of the $\mathrm{S} 0$ and $\mathrm{A} 0$ modes at $0.25 \mathrm{MHz}$ are shown in Fig. 8 as functions of the normalized crack length. For the numerical results, the transmission ratios of the $\mathrm{S} 0$ and $\mathrm{A} 0$ modes are relatively insensitive to the crack 
length when $C / W<0.3$ or $C / W>0.8$, which is a natural consequence since the excitation/detection areas as well as the main lobes of the incident wave have finite widths (c,f, Fig. 3). On the other hand, the transmission ratios decrease monotonically with the crack length in the intermediate range $0.3<C / W<$ 0.8 . The steeper gradient for the A0 mode may be accounted for by the narrower main lobe of the incident wave for that mode. It is shown in Fig. 8 that the slit-length dependence of the measured transmission ratios of both modes appears to follow the same trend as the numerical results. The discrepancy between the numerical and experimental results, particularly seen for the $\mathrm{A} 0$ mode when $C / W<0.3$ and $C / W>0.8$, may be due to the difference of the subtle side-lobe features of the incident wave profile between the analysis and experiment. Otherwise the numerical and experimental results are in excellent agreement, which indicates the effectiveness of the present numerical modeling in reproducing the Lamb wave measurements, particularly for the range $0.3<C / W<0.8$. It is noted again here that the crack-length dependence of the transmission ratios demonstrated here reflects the particular excitation/detection conditions and the relative crack location considered in this study, and the same holds true in the corresponding results for closed cracks discussed in the subsequent sections.

\subsection{Plate with a partially closed crack}

For the plate with a partially closed crack modeled as a spring-type interface in the present numerical simulation, the transmission waveforms of the S0 and A0 modes are shown in Fig. 9 for different interfacial stiffnesses and for the two crack lengths $C / W=0.5$ and 0.75 . With increasing interfacial stiffness, the transmission waveform of the S0 mode increases its magnitude and approaches the waveform for the reference plate $(C=0)$ as shown in Fig. 9(a) and (b). The transmission waveform of the A0 mode shows a similar trend for the shorter crack $(C / W=0.5$, Fig. $5(\mathrm{c}))$. In contrast, in the case of $C / W$ $=0.75$ shown in Fig. 9(d), the wave packet changes its shape significantly with increasing interfacial stiffness. The transmission ratios of the S0 and A0 modes are calculated from the amplitude spectra of the transmission waveforms based on Eq. (3) and shown in Fig. 10 as functions of the frequency for the two crack lengths. The transmission ratios of the S0 mode, shown in Fig. 10(a) and (b), are almost frequency-independent or slightly decreasing with the frequency in the range of $0.2 \mathrm{MHz}<f<0.4 \mathrm{MHz}$. On the other hand, the transmission ratios of the A0 mode, shown in Fig. 10(c) and (d), exhibit non-monotonic frequency dependence. The non-monotonic variation is more remarkable for the longer crack $(C / W=0.75)$, which exhibits a local minimum at $0.25 \mathrm{MHz}$ when $K_{\mathrm{N}}=0.0125 \mathrm{MPa} / \mathrm{nm}$ and at 0.28 $\mathrm{MHz}$ when $K_{\mathrm{N}}=0.02 \mathrm{MPa} / \mathrm{nm}$. Furthermore, the transmission ratios at $0.25 \mathrm{MHz}$ are shown in Fig. 11 as functions of the interfacial stiffness. The transmission ratio of the S0 mode increases monotonically with the interfacial stiffness for the two crack lengths. The transmission ratio of the A0 mode shows 
non-monotonic variation with the interfacial stiffness when $C / W=0.75$, with a local minimum of the transmission ratio at around $K_{\mathrm{N}}=0.01 \mathrm{MPa} / \mathrm{nm}$.

Figure 12 shows the transmission ratios of the $\mathrm{S} 0$ and $\mathrm{A} 0$ modes at $0.25 \mathrm{MHz}$ for partially closed cracks against their length, together with the corresponding results for the open crack. In the range where the crack length is sufficiently small (roughly for $C / W<0.3$ ), the transmission ratios of both modes remain close to unity irrespective of the interfacial stiffness, which is due to the finite width of the main lobes of the incident waves as discussed for open cracks. Beyond this range, the transmission ratios tend to decrease with increasing crack length, but show different variations depending on the interfacial stiffness. As the interfacial stiffness increases from zero, the crack-length dependence of the transmission ratio deviates from that for the open crack. A general trend is that the increase of the interfacial stiffness contributes to the increase of the transmission ratios. For the A0 mode, however, an exceptional behavior is observed when the crack is relatively long, i.e. $C / W>0.6$. Specifically, the transmission ratios for the stiffnesses $K_{\mathrm{N}}=0.005 \mathrm{MPa} / \mathrm{nm}$ and $0.0125 \mathrm{MPa} / \mathrm{nm}$ are smaller than the corresponding values for the open crack when the normalized crack length is 0.69 and 0.75 , respectively.

\subsection{Plate with a fatigue crack}

For the plate with the fatigue crack of total length $56 \mathrm{~mm}$ (EDM slit of $10 \mathrm{~mm}$ and fatigue crack 46 $\mathrm{mm}$ ), the measured waveforms for the S0 and A0 modes are shown in Fig. 13 for different levels of tensile loading, i.e., at the initial state (50 or $54 \mathrm{~N})$, at the maximally loaded state $(400 \mathrm{~N})$ and at the unloaded state $(0 \mathrm{~N})$. It is seen in Fig. 13 that the measured waveforms have lower magnitude at the maximally loaded state than at the unloaded state, for both S0 and A0 modes. The waveforms for the plate with the EDM slit of the same length $(56 \mathrm{~mm})$ are also shown in Fig. 13. For this plate, there was no visible difference in the measured waveforms for different levels of loading, so only the results at $0 \mathrm{~N}$ are shown.

The transmission ratios of the S0 and A0 modes were obtained from the amplitude spectra of the measured waveforms at three different frequencies of $0.2,0.25$ and $0.3 \mathrm{MHz}$. The results are shown in Fig. 14 against the tensile load applied to the plate, together with the corresponding results for the plate with the EDM slit of the same length. It is first observed that the transmission ratios for the plate with the slit are essentially independent of the tensile load for both S0 and A0 modes, as expected naturally (since the results for the loading and the unloading processes are indistinguishable, only the latter results are shown in Fig. 14). On the other hand, the transmission ratios for the plate with the fatigue crack change with the tensile load, which indicates that the contact condition of the crack surfaces was varied by the applied loading. 
In Fig. 14(a), the transmission ratios of the S0 mode for the three frequencies are around 0.2 at the initial load, and decrease with the increasing tensile load. This is consistent with physical intuition since the tensile load will reduce the effect of crack closure. The ratios become stationary for higher loading, say, beyond $200 \mathrm{~N}$. At unloading, the transmission ratios follow similar curves to the loading process down to the initial load. As the load is decreased further, the transmission ratios increase and take the values around 0.3 at $0 \mathrm{~N}$. It is also noted that the frequency does not have significant effect on the transmission ratio.

The tensile load dependence of the transmission ratio of the A0 mode is shown in Fig. 14(b). In contrast to the results of the S0 mode, the results for the A0 mode show different load dependence for different frequencies. At the higher frequency of $0.3 \mathrm{MHz}$, the transmission ratio shows monotonic dependence on the tensile load similarly to the case of the S0 mode, although it exhibits only slight change for the tensile load above the initial value. At the lower frequency of $0.2 \mathrm{MHz}$, the transmission ratio shows increasing behavior with increasing tension above the initial load and saturates for the load above around $200 \mathrm{~N}$. At unloading, the ratio follows the similar path down to the initial load, and increases slightly as the load approaches $0 \mathrm{~N}$. At the central frequency of $0.25 \mathrm{MHz}$, the transmission ratio shows intermediate characteristics between the two frequencies. The non-monotonic load dependence of the transmission ratio observed here for the $\mathrm{A} 0$ mode will be discussed in the next section.

\section{Discussions}

\subsection{Effect of interfacial stiffness and frequency on the Lamb wave transmission}

In the results of the numerical simulations demonstrated in Section 4.2, the qualitative nature of the Lamb wave interaction with a closed crack is remarkably different between the S0 and A0 modes. Namely, the transmission ratio of the S0 mode across the closed crack increases monotonically with the increasing interfacial stiffness of the crack. The ratio decreases monotonically with the frequency, although its variation is not significant in the range demonstrated in the present analysis. Such monotonic dependence of the wave transmission on the interfacial stiffness and the frequency has also been observed for bulk ultrasonic waves transmitted across a spring-type interface [33, 35]. In contrast, the numerical results have demonstrated that the transmission ratio of the A0 mode across the closed crack exhibits non-monotonic dependence on the interfacial stiffness and the frequency, featured by a local minimum at a certain value of these parameters as shown in Figs. 10-12.

In the foregoing study, Mori et al. [26] presented a theoretical analysis of the reflection and transmission of Lamb waves across a spring-type joint interface of two plates. Their two-dimensional analysis corresponds to the situation in which the plate width and the crack length are infinitely long and the plate is subjected to the incidence of the pure S0 or A0 mode. They obtained similar findings regarding 
the effect of the interfacial stiffness and the frequency on the transmission characteristics of the S0 and A0 modes. Based on the analysis of Mori et al. [26], the reason behind the qualitative difference between the $\mathrm{S} 0$ and $\mathrm{A} 0$ modes can be physically explained in the following manner. When the S0 mode is incident on the spring-type interface of plates, not only the reflected and transmitted S0 mode but also other symmetric Lamb modes $(\mathrm{S} 1, \mathrm{~S} 2, \ldots)$ can be generated at the interface (due to the symmetry of the interface boundary conditions only the symmetric modes are generated for the incidence of a symmetric mode). In the frequency range examined here, these higher-order modes are non-propagating and localized near the interface. According to the analysis [26], their magnitudes are negligible as compared to the magnitude of the incident S0 mode. Therefore, the reflection/transmission of the S0 mode is similar to the bulk longitudinal wave normally reflected/transmitted at the spring-type interface. This explains the monotonic dependence of the S0 mode transmission on the interfacial stiffness and the frequency.

When the A0 mode is incident on the interface, other antisymmetric and localized Lamb modes (A1, $\mathrm{A} 2, \ldots)$ can be generated likewise. A key feature is, however, that the magnitude of the A1 mode is of comparable order to the incident $\mathrm{A} 0$ mode while the other antisymmetric modes $(\mathrm{A} 2, \ldots)$ are of negligible magnitudes. Namely, the interface boundary conditions are essentially met by the reflected/transmitted A0 modes as well as the localized A1 modes on both sides of the interface. Since these two modes are involved in the reflection/transmission process with their magnitude varying with the interfacial stiffnesses and the frequency, the monotonic dependence as in the case of the S0 mode incidence is no longer expected. In particular, at a certain frequency for given interfacial stiffnesses (or at certain interfacial stiffnesses for a given frequency), the wave motion on the transmission side can be essentially supported only by the non-propagating A1 mode, which gives vanishing transmission of the A0 mode. The through-thickness profiles of the S0, A0, S1 and A1 Lamb modes at $0.25 \mathrm{MHz}$ are shown in Fig. 15. Clearly, the S0 mode is predominantly associated with the in-plane motion, while the A0 mode is dominated by the out-of-plane motion. It can be noted that the profile of the A1 mode resembles that of the A0 mode to some extent, although the in-plane motion is dominant for the A1 mode. This indicates that the A0 mode may be easily coupled with the A1 mode at the interface, which appears to support the above reasoning regarding the role played by the $\mathrm{A} 1$ mode in the reflection/transmission of the A0 mode.

\subsection{Examination of experimental results based on the spring-type interface model}

In the experimental results shown in Fig. 14(a), the transmission ratio of the S0 mode decreases monotonically as the tensile load is increased. Qualitatively, this is consistent with the monotonically increasing variation of the transmission ratio with the interfacial stiffness found in the numerical analysis, since the tensile load tends to open the crack, which theoretically corresponds to the reduction of its 
interfacial stiffnesses. The experimental results for the S0 mode in Fig. 14(a) also show that the transmission ratio is not sensitive to the frequency, which qualitatively corresponds to the feature found in the numerical results in Fig. 10. Rigorously speaking, however, the measured transmission ratio is slightly increasing with the frequency in Fig. 14(a), while the numerical result in Fig. 10 is decreasing with it. This may partly be attributable to the inhomogeneous nature of rough-surface contact, which yields higher interfacial stiffnesses, hence higher transmission, for higher frequencies as discussed in Refs. [23, 33] for aluminum alloy specimens and in Refs. $[36,37]$ for fractures in geophysics. It should be noted, however, that the theoretical analysis assumes constant interfacial stiffnesses which are uniform on the crack surfaces and have the constant ratio $K_{\mathrm{T}} / K_{\mathrm{N}}=0.3$. Such an assumption is not guaranteed for the specimen used in the measurement, so the quantitative comparison is out of the scope of the present study from the outset.

In Fig. 14(b), the transmission ratio of the A0 mode across the fatigue crack obtained in the experiment shows complicated effects of the tensile load and the frequency. Specifically, the tensile loading, which should weaken the crack closure, does not necessarily lead to the reduction of the transmission ratio. This complex behavior is also observed numerically in Fig. 11 as the non-monotonic influence of the interfacial stiffness on the transmission ratio. In order to examine the experimental results for the fatigue crack in more detail, the numerical simulation of the Lamb wave transmission was performed using the spectral element method by assuming the same crack length as the experiment. Namely, the crack examined in the experiment, which is a $46 \mathrm{~mm}$-long fatigue crack grown from a $10 \mathrm{~mm}$-long EDM slit, was modeled as the crack with the open part $10 \mathrm{~mm}$ and the closed part $46 \mathrm{~mm}$. The computed transmission ratios of the $\mathrm{S} 0$ and $\mathrm{A} 0$ modes are shown in Fig. 16 as functions of the interfacial stiffness. It is noted again that the information of the distribution of the interfacial stiffnesses on the fatigue crack surfaces in the experiment was not available, so the interfacial stiffnesses were assumed to be uniform on the crack surfaces with the constant ratio $K_{\mathrm{T}} / K_{\mathrm{N}}=0.3$. This simplified modeling makes the quantitative comparison between the experimental and numerical results less meaningful, so the discussion is restricted to the qualitative comparison. In order to do this in a physically intuitive manner, the inverse of $K_{\mathrm{N}}$ is taken along the horizontal axis of Fig. 16. In Fig. 16(a), the transmission ratio of the S0 mode decreases with increasing $1 / K_{\mathrm{N}}$ (decreasing $K_{\mathrm{N}}$ ), and the frequency dependence is not significant. On the other hand, in Fig. 16(b), the transmission ratio of the A0 mode shows different behavior depending on the frequency. At $0.3 \mathrm{MHz}$ the ratio decreases monotonically with $1 / K_{\mathrm{N}}$ in the entire range plotted, while at $0.2 \mathrm{MHz}$ the ratio first decreases with $1 / K_{\mathrm{N}}$ but turns to increase with it for higher values of $1 / K_{\mathrm{N}}$ (for lower $K_{\mathrm{N}}$ ). The inverse stiffness $1 / K_{\mathrm{N}}$ can be regarded as a measure of the tensile loading applied to the closed crack. It is interesting to note that if the fatigue crack had a certain finite interfacial stiffness, say $1 / K_{\mathrm{N}}=3 \times 10^{2}$ 
$\mathrm{nm} / \mathrm{MPa}$, in the unloaded state, the variation of the transmission ratio of both $\mathrm{S} 0$ and $\mathrm{A} 0$ modes with increasing $1 / K_{\mathrm{N}}$ would qualitatively reproduce the load dependence in the experimental results in Fig. 14 .

\section{Concluding remarks}

In this study, the transmission characteristics of the S0 and A0 Lamb waves across a through-thickness crack have been investigated numerically and experimentally. The three-dimensional Lamb wave propagation in a plate with an open or a partially closed crack has been analyzed by the spectral element method by modeling the partially closed crack as a spring-type interface characterized by normal and tangential stiffnesses. The numerical analysis has shown that the transmission ratio of the S0 mode increases monotonically with increasing interfacial stiffness, but the transmission ratio of the A0 mode varies in a complicated manner depending on the interfacial stiffness and the frequency. The Lamb wave transmission measurements have also been performed for aluminum alloy plates with an EDM slit and for an aluminum alloy plate with a fatigue crack. For the plates with a slit, the experimental results are in reasonable agreement with the numerical analysis for open cracks for both $\mathrm{S} 0$ and A0 modes. For the plate with a fatigue crack, the tensile load reduces the transmission ratio of the S0 mode, but its effect on that of the A0 mode is not monotonic and depends on the frequency. Features qualitatively corresponding with the experimental results have been shown by the numerical analysis for the closed crack based on the spring-type interface model.

The present study has shown that the Lamb wave interaction with a crack is significantly influenced by the extent of its partial closure. Moreover, the crack closure has different effects on different Lamb modes. The transmission ratio of the S0 mode is increased by the crack closure. On the other hand, the crack closure influences the transmission of the A0 mode in a more complicated manner, i.e., the magnitude of the transmitted signal of the A0 mode cannot be simply related to the state of crack closure. These features should be properly accounted for in the monitoring of fatigue crack growth using Lamb waves. One remedy in practical applications would be that the crack be monitored using the S0 mode Lamb wave while it is subjected to tensile loading to make it fully open. Further explorations would facilitate an effective Lamb wave-based characterization method for closed cracks. The numerical simulations for closed cracks based on the spring-type interface model will offer a convenient tool to examine the feasibility of different characterization techniques for fatigue cracks. 


\section{Acknowledgement}

This work has been supported by JSPS KAKENHI Grant Numbers 26-2190 and JP16H04235. The authors are grateful to the anonymous reviewers for their useful and constructive comments to our manuscript.

\section{References}

[1] K. F. Graff, Wave Motion in Elastic Solids, 1975, Oxford University Press, London.

[2] J. L. Rose, Ultrasonic Waves in Solid Media, 1999, Cambridge University Press, Cambridge.

[3] M. Koshiba, S. Karakida, M. Suzuki, Finite-element analysis of Lamb wave scattering in an elastic plate waveguide, IEEE Trans. Sonics Ultrason. 31 (1984) 18-25.

[4] D. Alleyne, P. Cawley, The interaction of Lamb waves with defects, IEEE Trans. Ultrason. Ferr. 39 (1992) 381-397.

[5] Y. Cho, D. D. Hongerholt, J. L. Rose, Lamb wave scattering analysis for reflector characterization, IEEE Trans. Ultrason. Ferroelectr. Freq. Control 44 (1997) 44-52.

[6] M. J. S. Lowe, O. Diligent, Low-frequency reflection characteristics of the s0 Lamb wave from a rectangular notch in a plate, J. Acoust. Soc. Am. 111 (2002) 64-74.

[7] M. Castaings, E. Le Clezio, B. Hosten, Modal decomposition method for modeling the interaction of Lamb waves with cracks, J. Acoust. Soc. Am. 112 (2002) 2567-2582.

[8] Z. Chang, A. Mal, Scattering of Lamb waves from a rivet hole with edge cracks, Mech. Mater. 31 (1999) 197-204.

[9] Y. Lu, L. Ye, Z. Su, C. Yang, Quantitative assessment of through-thickness crack size based on Lamb wave scattering in aluminium plates, NDT \& E Int. 41 (2008) 59-68.

[10] L. Yu, C. A. C. Leckey, Lamb wave-based quantitative crack detection using a focusing array algorithm, J. Intell. Mater. Syst. Struct. 24 (2012) 1138-1152.

[11] S. Grondel, C. Delebarre, J. Assaad, J.-P. Dupuis, L. Reithler, Fatigue crack monitoring of riveted aluminium strap joints by Lamb wave analysis and acoustic emission measurement techniques, NDT \& E Int. 35 (2002) 137-146.

[12] J.-B. Ihn, F.-K. Chang, Detection and monitoring of hidden fatigue crack growth using a built-in piezoelectric sensor/actuator network: I. Diagnostics, Smart Mater. Struct. 13 (2004) 609-620.

[13] J.-B. Ihn, F.-K. Chang, Detection and monitoring of hidden fatigue crack growth using a built-in piezoelectric sensor/actuator network: II. Validation using riveted joints and repair patches, Smart Mater. Struct. 13 (2004) 621-630. 
[14] W. H. Leong, W. J. Staszewski, B. C. Lee, F. Scarpa, Structural health monitoring using scanning laser vibrometry: III. Lamb waves for fatigue crack detection, Smart Mater. Struct. 14 (2005) $1387-1395$.

[15] J.-B. Ihn, F.-K. Chang, Pitch-catch active sensing methods in structural health monitoring for aircraft structures, Struct. Health Monit. 7 (2008) 5-19.

[16] B. C. Lee, W. J. Staszewski, Modelling of Lamb wave interaction with open and closed fatigue cracks for damage detection, IOP Conf. Ser.: Mater. Sci. Eng. 10 (2010) 012059.

[17] H. Cho and C. J. Lissenden, Structural health monitoring of fatigue crack growth in plate structures with ultrasonic guided waves, Struct. Health Monit. 11 (2011) 393-404.

[18] O. Buck, R. B. Thompson, D. K. Rehbein, The interaction of ultrasound with contacting asperities: Applications to crack closure and fatigue crack growth, J. Nondestr. Eval. 4 (1984) 203-212.

[19] M. A. S. Akanda, M. Saka, Relationship between closure stress of small fatigue crack and ultrasonic response, J. Nondestr. Eval. 23 (2004), 37-47.

[20] K. Makino, S. Biwa, H. Sakamoto, J. Yohso, Ultrasonic evaluation of fatigue cracks at the wheel seat of a miniature wheelset, Nondestr. Test. Eval. 27 (2012) 29-46.

[21] Y. Shen, C. E. S. Cesnik, Modeling of nonlinear interactions between guided waves and fatigue cracks using local interaction simulation approach, Ultrasonics 74 (2017) 106-123.

[22] Y. Yang, C.-T. Ng, A. Kotousov, H. Sohn, H. J. Lim, Second harmonic generation at fatigue cracks by low-frequency Lamb waves: Experimental and numerical studies, Mech. Sys. Signal Processing 99 (2018) 760-773.

[23] N. Mori, S. Biwa, Transmission characteristics of the S0 and A0 Lamb waves at contacting edges of plates, Ultrasonics 81 (2017) 93-99.

[24] A. Boström, G. Wickham, On the boundary conditions for ultrasonic transmission by partially closed cracks, J. Nondestr. Eval. 10 (1991) 139-149.

[25] P. B. Nagy, Ultrasonic classification of imperfect interfaces, J. Nondestr. Eval. 11 (1992) 127-139.

[26] N. Mori, S. Biwa, T. Hayashi, Reflection and transmission of Lamb waves at an imperfect joint of plates, J. Appl. Phys. 113 (2013) 074901.

[27] A. T. Patera, A spectral element method for fluid dynamics: Laminar flow in a channel expansion, J. Comput. Phys. 54 (1984) 468-488.

[28] P. Kudela, A. Zak, M. Krawczuk, W. Ostachowicz, Modeling of wave propagation in composite plates using the time domain spectral element method, J. Sound Vib. 302 (2007) 728-745.

[29] Y. Kim, S. Ha, F.-K. Chang, Time-domain spectral element method for built-in piezoelectric-actuator-induced Lamb wave propagation analysis, AIAA J. 46 (2008) 591-600. 
[30] H. Peng, G. Meng, F. Li, Modeling of wave propagation in plate structures using three-dimensional spectral element method for damage detection, J. Sound Vib. 320 (2009) 942-954.

[31] A. Baltazar, S. I. Rokhlin, C. Pecorari, On the relationship between ultrasonic and micromechanical properties of contacting rough surfaces, J. Mech. Phys. Solids 50 (2002) 1397-1416.

[32] D. Liaptsis, B. Drinkwater, R. Thomas, The interaction of oblique incidence ultrasound with rough, partially contacting interfaces, Nondestr. Test. Eval. 21 (2006) 109-121.

[33] S. Biwa, S. Hiraiwa, E. Matsumoto, Stiffness evaluation of contacting surfaces by bulk and interface waves, Ultrasonics 47 (2007) 123-129.

[34] S. Biwa, S. Hiraiwa, E. Matsumoto, Pressure-dependent stiffnesses and nonlinear ultrasonic response of contacting surfaces, J. Solid Mech. Mater. Eng. 3 (2009) 10-21.

[35] B. W. Drinkwater, R. S. Dwyer-Joyce, P. Cawley, A study of the interaction between ultrasound and a partially contacting solid-solid interface, Proc. R. Soc. London, Ser. A 452 (1996) 2613-2628.

[36] L. Prrak-Nolte, D. D. Nolte, Frequency dependence of fracture stiffness, Geophys. Res. Lett. 19 (1992) 325-328.

[37] A. F. Baird, J.-M. Kendall, D. A. Angus, Frequency-dependent seismic anisotropy due to fractures: fluid flow versus scattering, Geophysics 78 (2013) WA111-WA122. 
Table 1 The phase velocity $c_{\mathrm{p}}$ and group velocity $c_{\mathrm{g}}$ of the $\mathrm{S} 0$ and $\mathrm{A} 0 \mathrm{Lamb}$ modes.

\begin{tabular}{cc|cc}
\hline Frequency $[\mathrm{MHz}]$ & Mode & $c_{\mathrm{p}}[\mathrm{km} / \mathrm{s}]$ & $c_{\mathrm{g}}[\mathrm{km} / \mathrm{s}]$ \\
\hline 0.25 & S0 & 5.28 & 5.00 \\
0.25 & A0 & 2.47 & 3.20
\end{tabular}


Figure legends

Fig. 1 Schematic of an elastic plate with a through-thickness crack.

Fig. 2 (a) Phase and (b) group velocities of guided waves in an aluminum plate of thickness $5 \mathrm{~mm}$.

Fig. 3 Distribution of the out-of-place displacement on the upper surface of the plate and its transverse profile at $x=-30 \mathrm{~mm}$ for (a) $\theta=31 \mathrm{deg}$ (S0 mode incidence) at elapsed time $t=22.5 \mu \mathrm{s}$ and (b) $\theta=75$ $\operatorname{deg}$ (A0 mode incidence) at elapsed time $t=31.0 \mu$ s. The displacement is normalized by the maximum of the transverse profile.

Fig. 4 Relation between the transmission coefficients and the interfacial stiffness for the S0 and A0 modes at $0.25 \mathrm{MHz}$, obtained by the present analysis and by the two-dimensional analysis.

Fig. 5 Schematic of the aluminum alloy plate specimens used in the experiment, (a) specimen with an EDM slit of different lengths $C$, (b) specimen with a fatigue crack grown from an EDM slit.

Fig. 6 Schematic of the experimental setup.

Fig. 7 Calculated transmission waveforms of the (a) S0 and (b) A0 modes and the measured transmission waveforms of the (c) S0 and (d) A0 modes for open cracks of different lengths $C / W$.

Fig. 8 Variation of the calculated and measured transmission ratios of the $\mathrm{S} 0$ and $\mathrm{A} 0$ modes at $0.25 \mathrm{MHz}$ with the length of the open crack $C / W$.

Fig. 9 Calculated transmission waveforms of the S0 mode for closed cracks of the lengths (a) $C / W=0.5$ and (b) $C / W=0.75$ and of the A0 mode for (c) $C / W=0.5$ and (d) $C / W=0.75$, for different interfacial stiffnesses.

Fig. 10 Frequency dependence of the calculated transmission ratio of the S0 mode for closed cracks of the lengths (a) $C / W=0.5$ and (b) $C / W=0.75$ and of the $\mathrm{A} 0$ mode for (c) $C / W=0.5$ and (d) $C / W=$ 0.75 , for different interfacial stiffnesses.

Fig. 11 Variation of the calculated transmission ratios of the $\mathrm{S} 0$ and $\mathrm{A} 0$ modes at $0.25 \mathrm{MHz}$ with the interfacial stiffness for closed cracks of the lengths $C / W=0.5$ and $C / W=0.75$.

Fig. 12 Variation of the calculated transmission ratios of the (a) S0 and (b) A0 modes at $0.25 \mathrm{MHz}$ with the length of the partially closed crack $C / W$ for different interfacial stiffnesses.

Fig. 13 Measured transmission waveforms of the (a) S0 and (b) A0 modes for the plate with a fatigue crack for different tensile loads. 
Fig. 14 Variation of the measured transmission ratios of the (a) S0 and (b) A0 modes with the tensile load at different frequencies for the plate with a fatigue crack and for the plate with an EDM slit.

Fig. 15 Through-thickness displacement profiles for the (a) S0, (b) A0, (c) S1 and (d) A1 modes at 0.25 MHz.

Fig. 16 Variation of the calculated transmission ratios of the (a) S0 and (b) A0 modes with the inverse of the interfacial stiffness $1 / K_{\mathrm{N}}$ at different frequencies. 


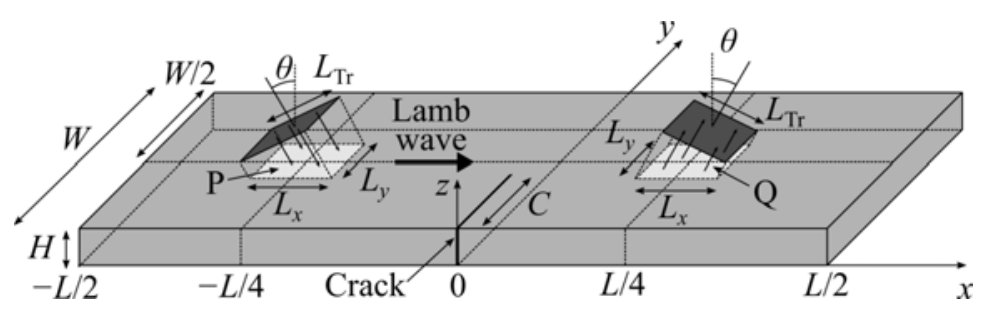

Fig. 1 Schematic of an elastic plate with a through-thickness crack.

(a)

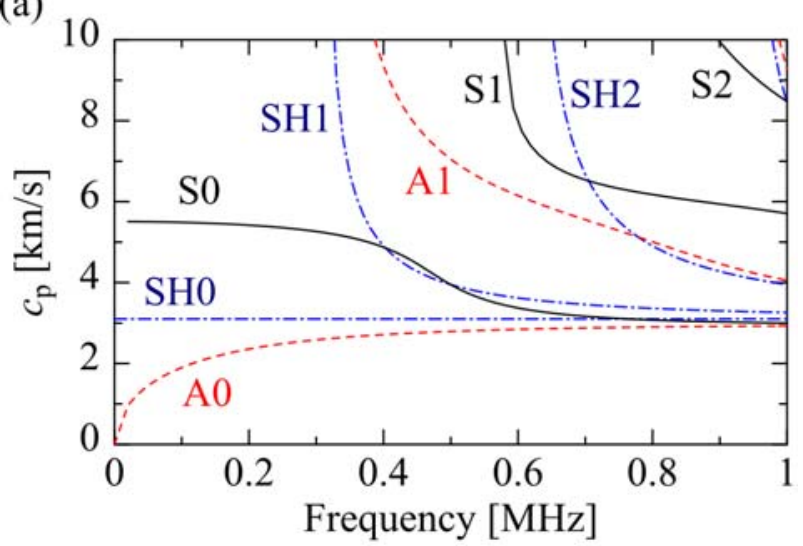

(b)

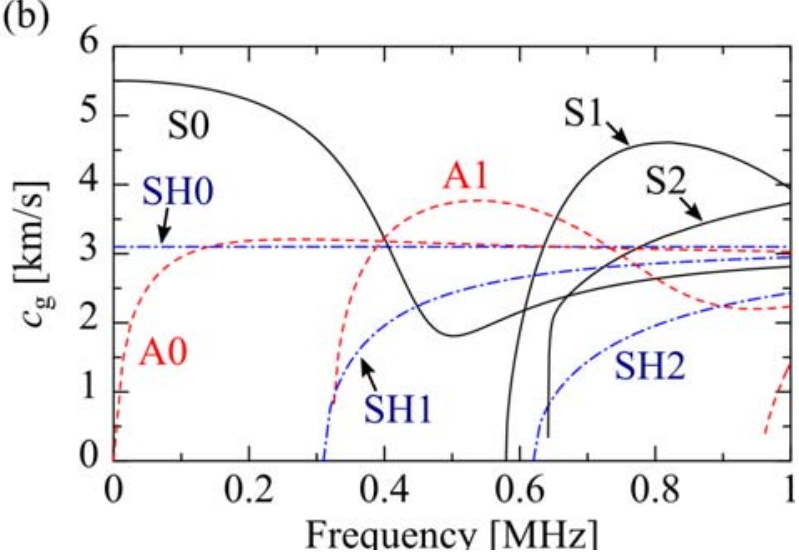

Fig. 2 (a) Phase and (b) group velocities of guided waves in an aluminum plate of thickness $5 \mathrm{~mm}$. 
(a)
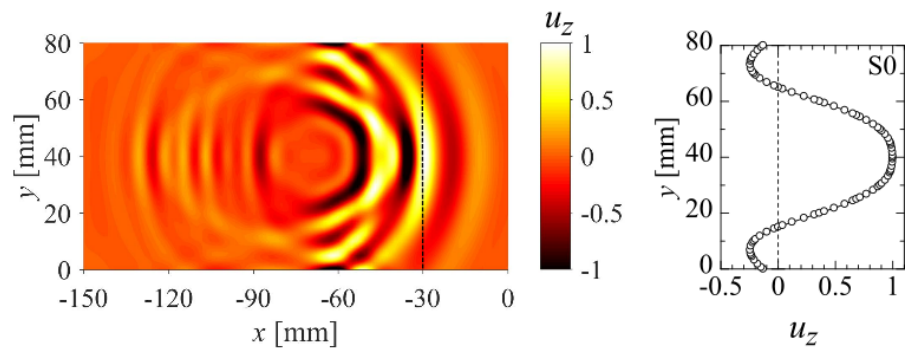

(b)
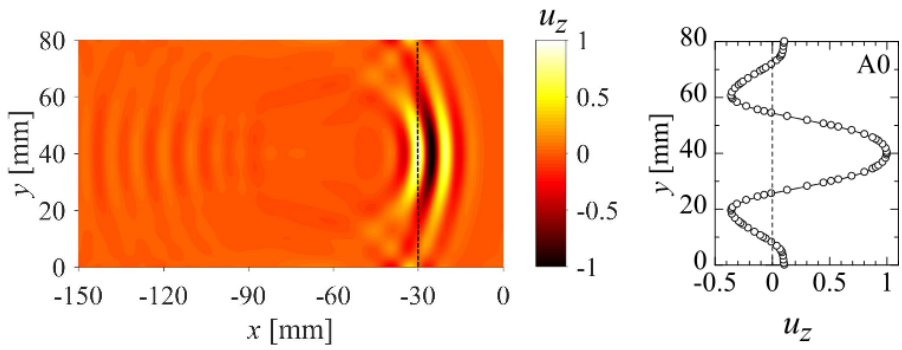

Fig. 3 Distribution of the out-of-place displacement on the upper surface of the plate and its transverse profile at $x=-30 \mathrm{~mm}$ for (a) $\theta=31 \mathrm{deg}$ ( $\mathrm{S} 0$ mode incidence) at elapsed time $t=22.5 \mu \mathrm{s}$ and (b) $\theta=75$ $\operatorname{deg}$ (A0 mode incidence) at elapsed time $t=31.0 \mu \mathrm{s}$. The displacement is normalized by the maximum of the transverse profile.

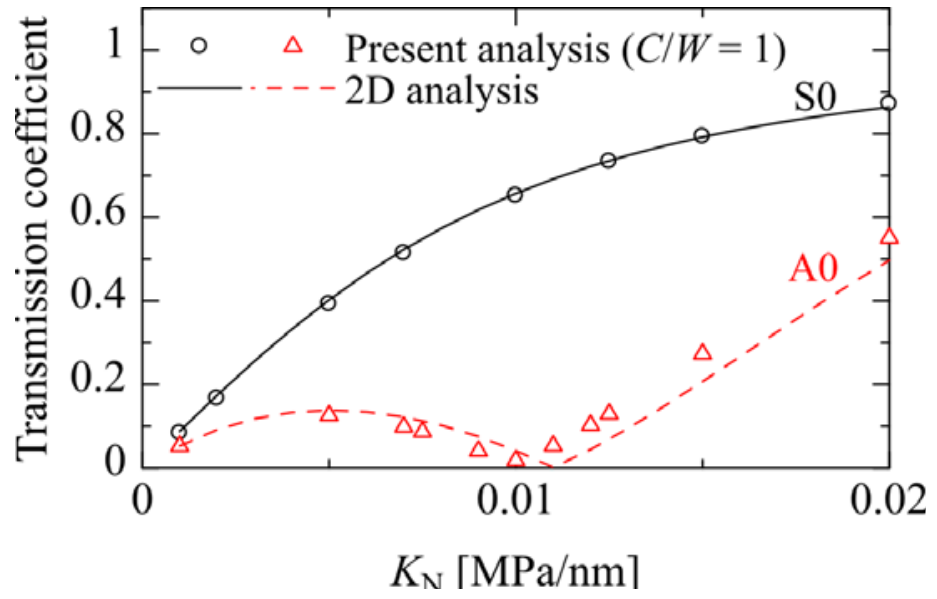

Fig. 4 Relation between the transmission coefficients and the interfacial stiffness for the S0 and A0 modes at $0.25 \mathrm{MHz}$, obtained by the present analysis and by the two-dimensional analysis. 

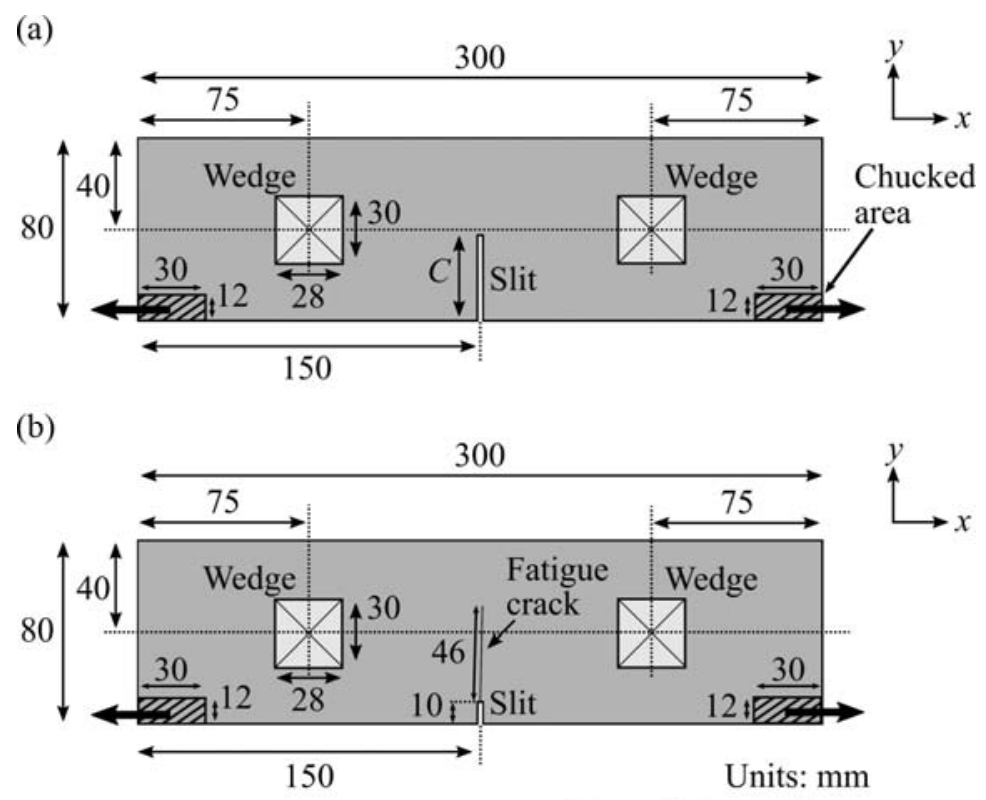

Plate thickness: $5 \mathrm{~mm}$

Fig. 5 Schematic of the aluminum alloy plate specimens used in the experiment, (a) specimen with an EDM slit of different lengths $C$, (b) specimen with a fatigue crack grown from an EDM slit.

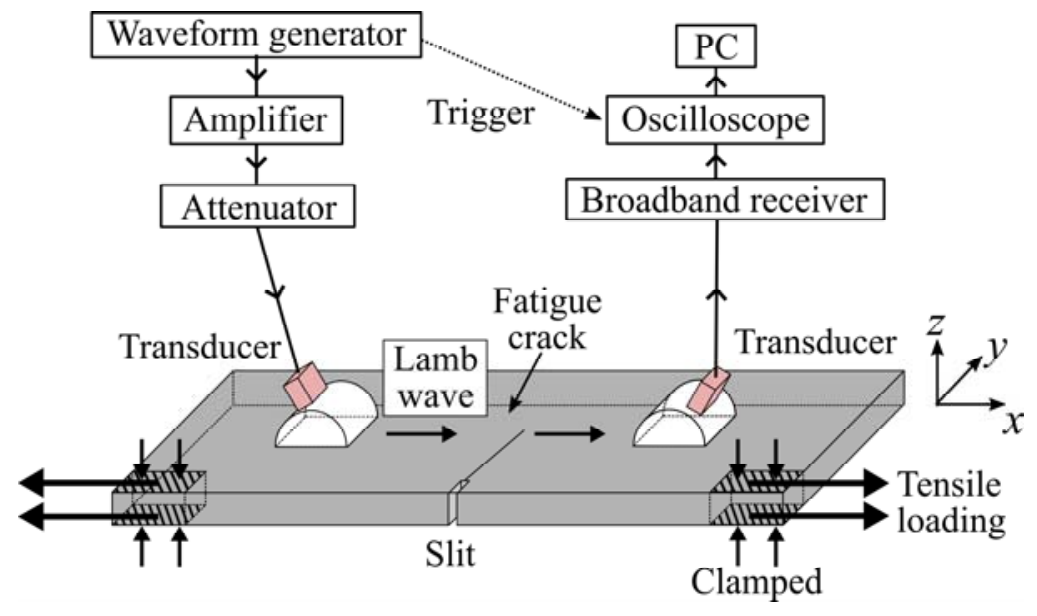

Fig. 6 Schematic of the experimental setup. 

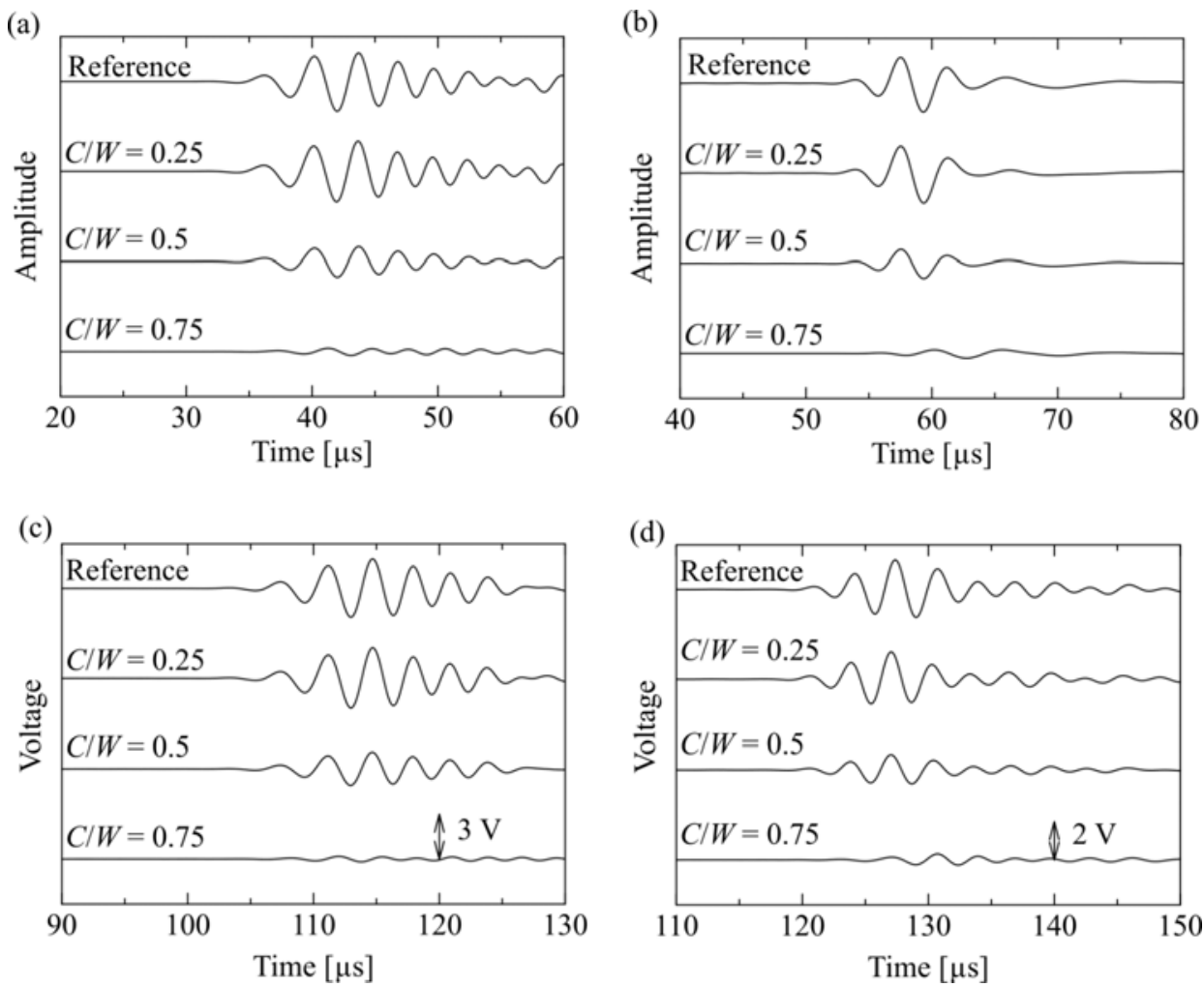

Fig. 7 Calculated transmission waveforms of the (a) S0 and (b) A0 modes and the measured transmission waveforms of the (c) S0 and (d) A0 modes for open cracks of different lengths $C / W$. 
(a)

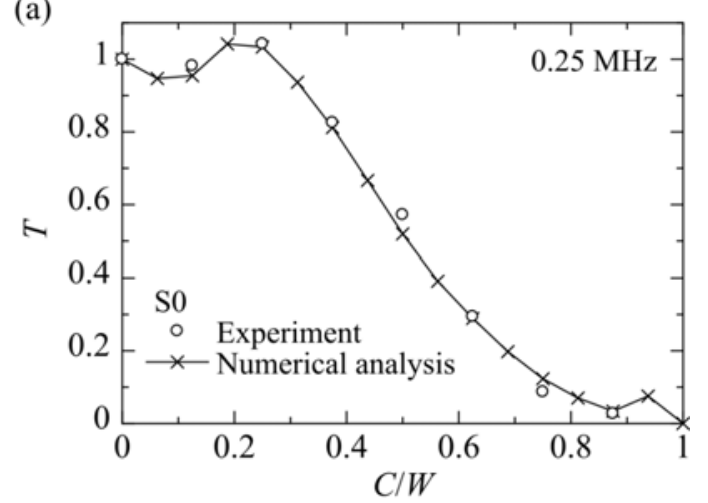

(b)

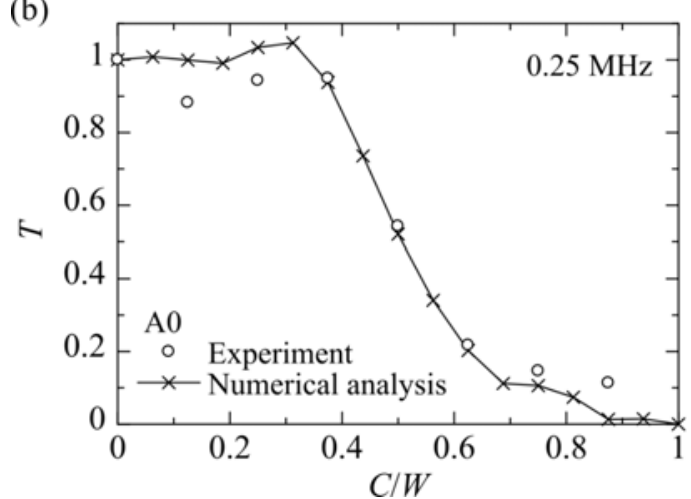

Fig. 8 Variation of the calculated and measured transmission ratios of the S0 and A0 modes at $0.25 \mathrm{MHz}$ with the length of the open crack $C / W$. 

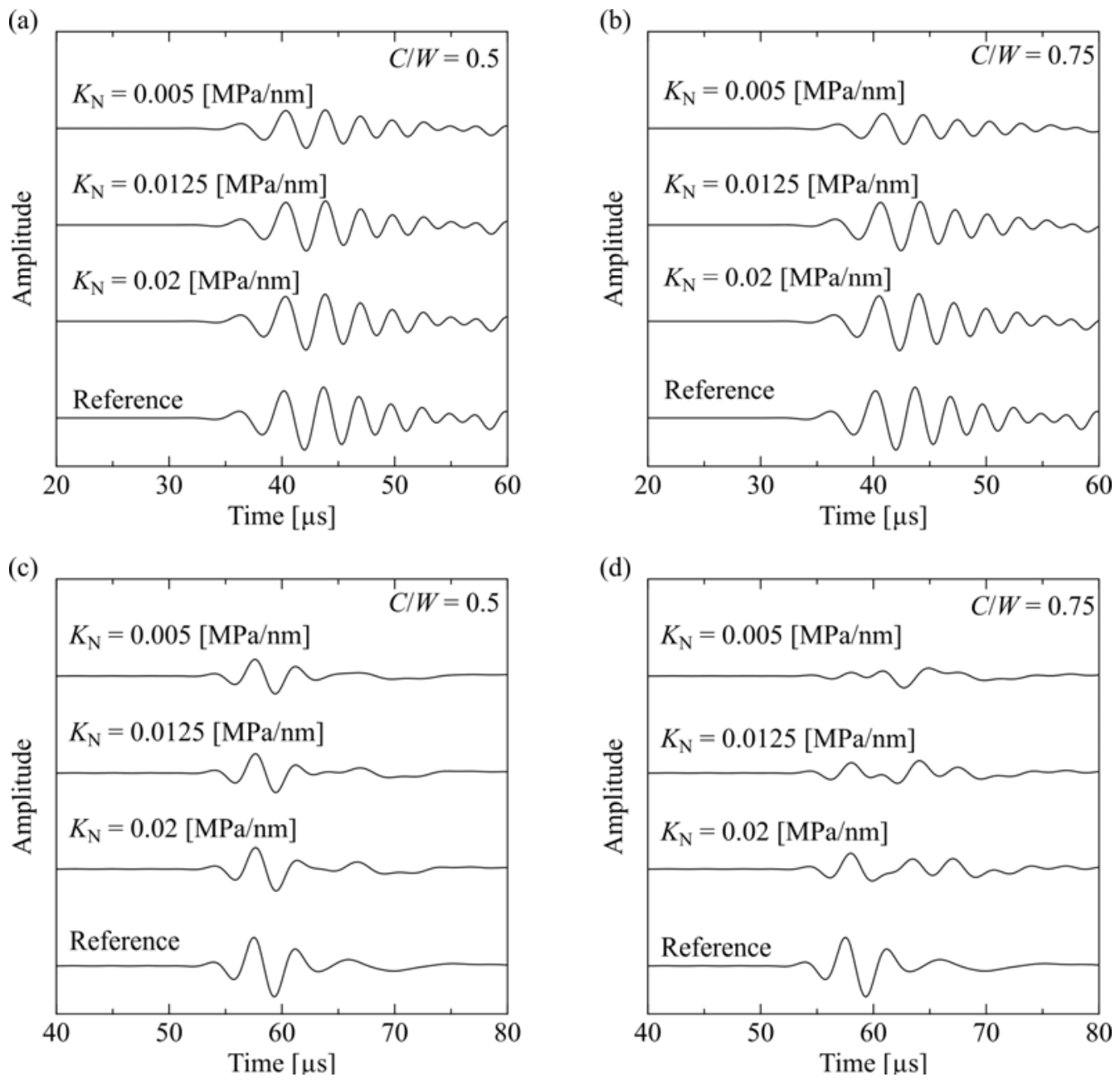

Fig. 9 Calculated transmission waveforms of the S0 mode for closed cracks of the lengths (a) $C / W=0.5$ and (b) $C / W=0.75$ and of the A0 mode for (c) $C / W=0.5$ and (d) $C / W=0.75$, for different interfacial stiffnesses. 
(a)

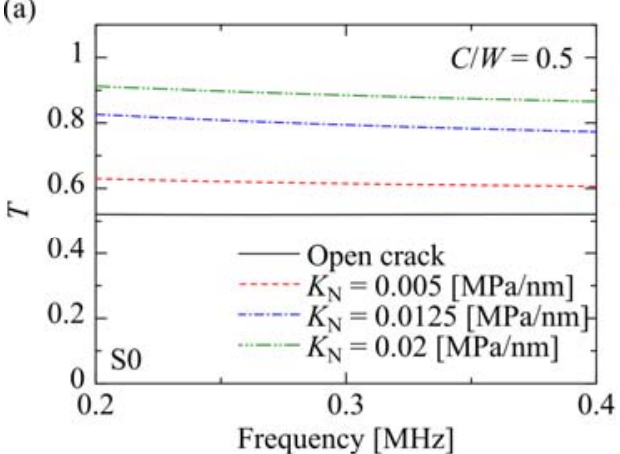

(c)

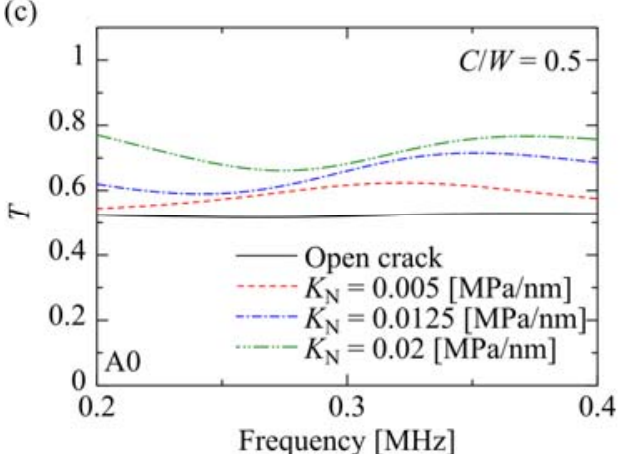

(b)

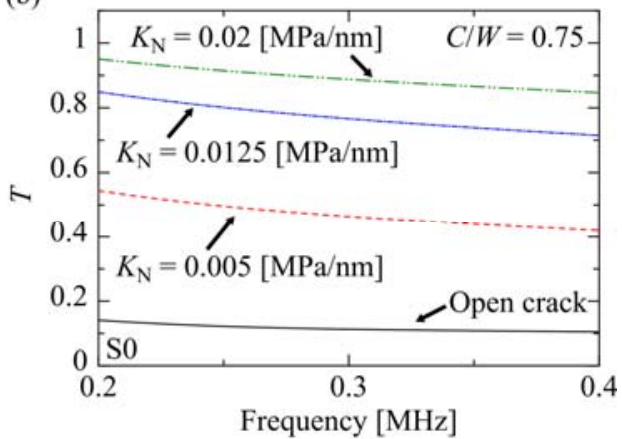

(d)

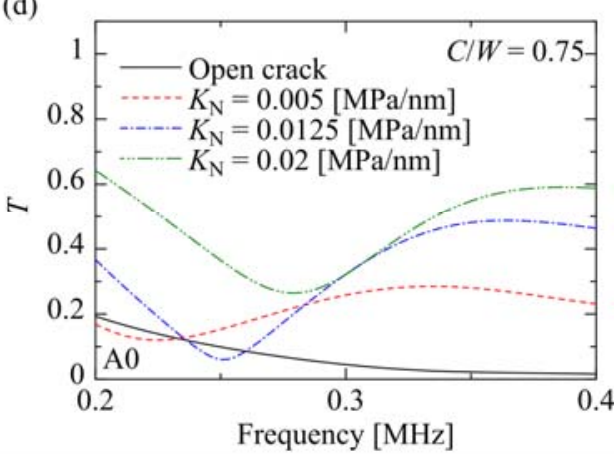

Fig. 10 Frequency dependence of the calculated transmission ratio of the S0 mode for closed cracks of the lengths (a) $C / W=0.5$ and (b) $C / W=0.75$ and of the A0 mode for (c) $C / W=0.5$ and (d) $C / W=$ 0.75 , for different interfacial stiffnesses.

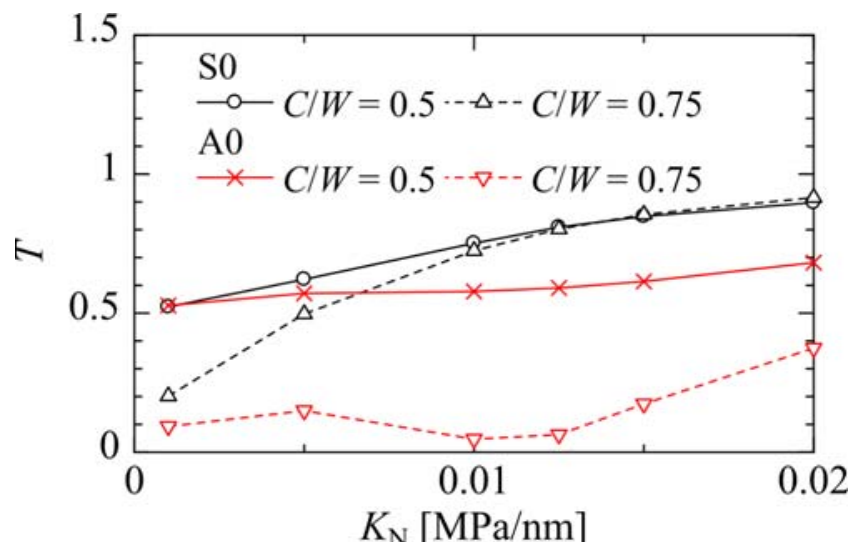

Fig. 11 Variation of the calculated transmission ratios of the $\mathrm{S} 0$ and $\mathrm{A} 0$ modes at $0.25 \mathrm{MHz}$ with the interfacial stiffness for closed cracks of the lengths $C / W=0.5$ and $C / W=0.75$. 
(a)

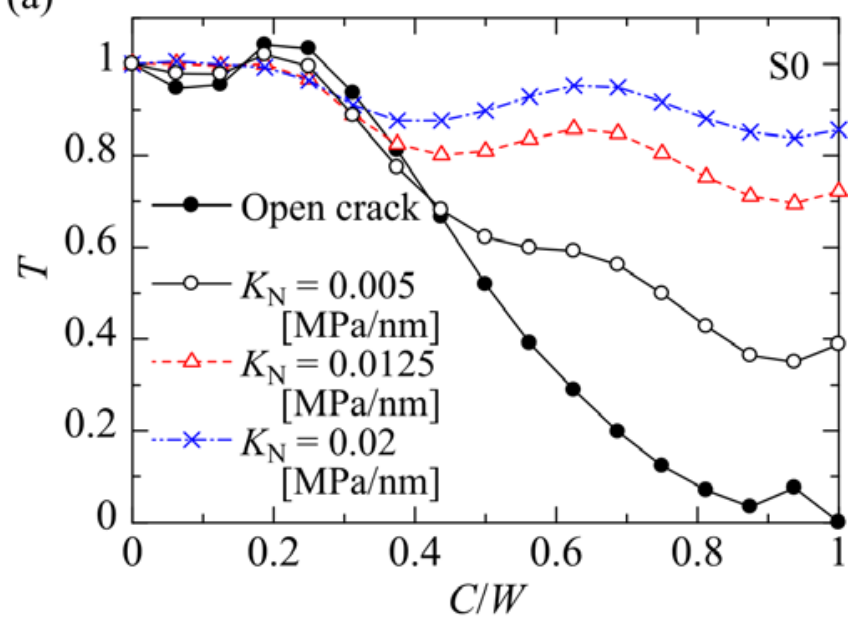

(b)

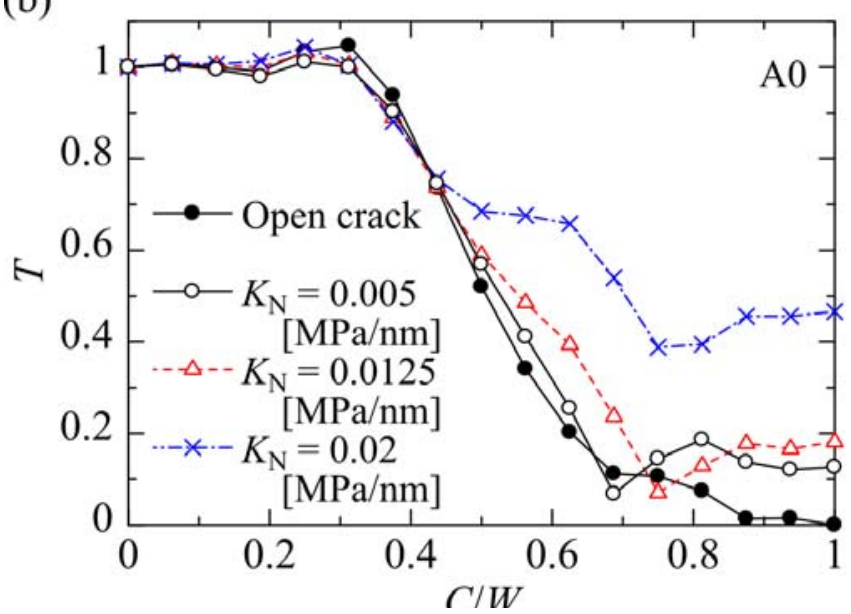

Fig. 12 Variation of the calculated transmission ratios of the (a) S0 and (b) A0 modes at $0.25 \mathrm{MHz}$ with the length of the partially closed crack $C / W$ for different interfacial stiffnesses. 

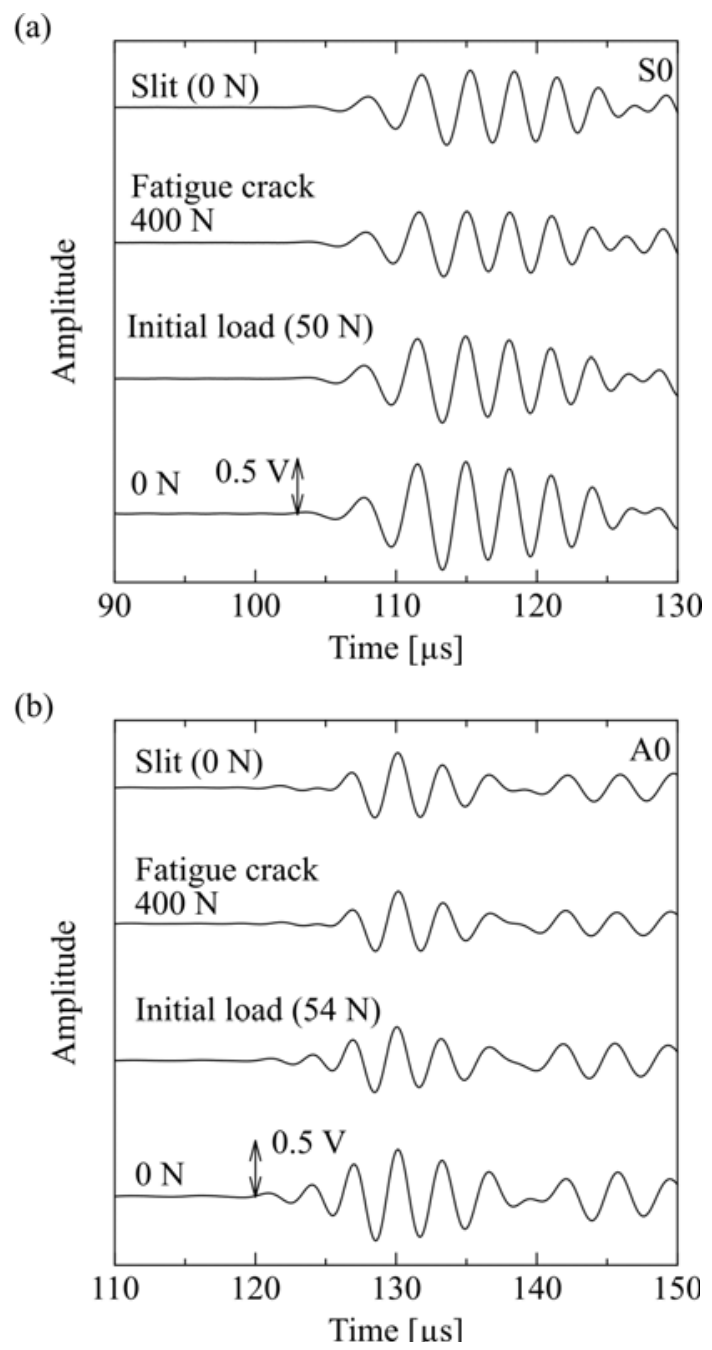

Fig. 13 Measured transmission waveforms of the (a) S0 and (b) A0 modes for the plate with a fatigue crack for different tensile loads. 
(a)

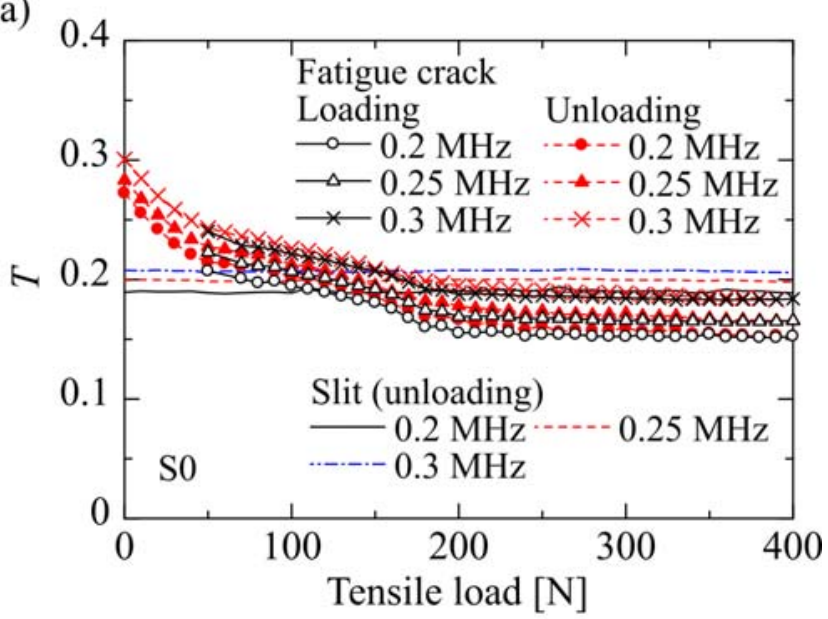

(b)

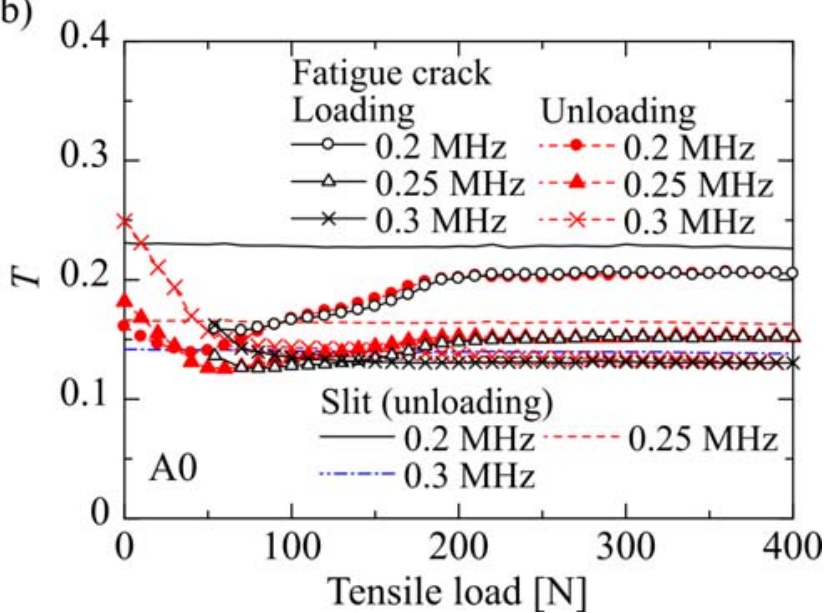

Fig. 14 Variation of the measured transmission ratios of the (a) S0 and (b) A0 modes with the tensile load at different frequencies for the plate with a fatigue crack and for the plate with an EDM slit. 
(a)

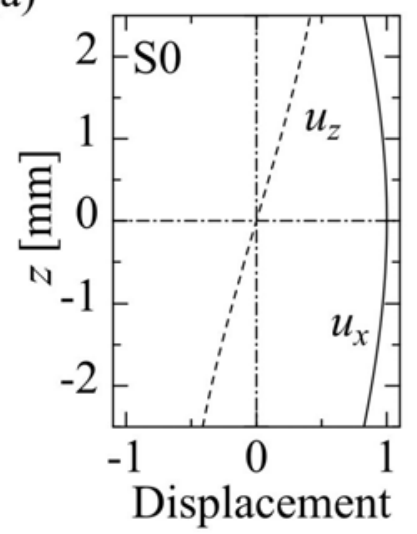

(c)

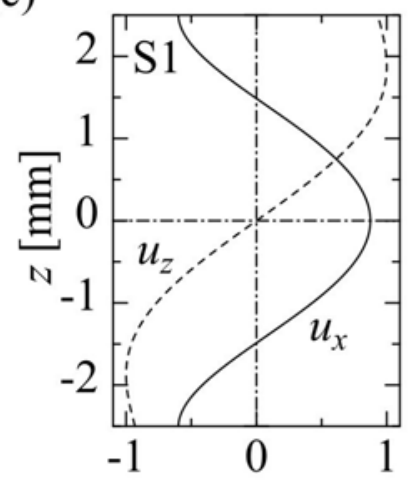

Displacement (b)

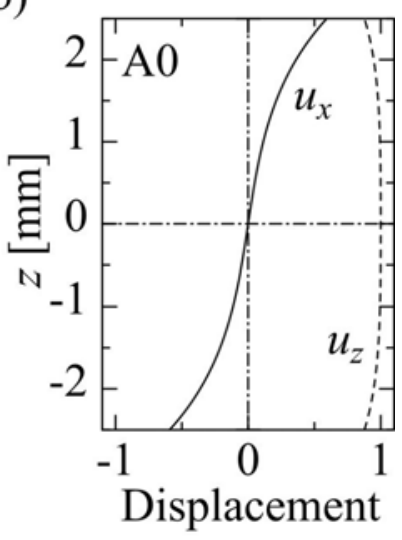

(d)

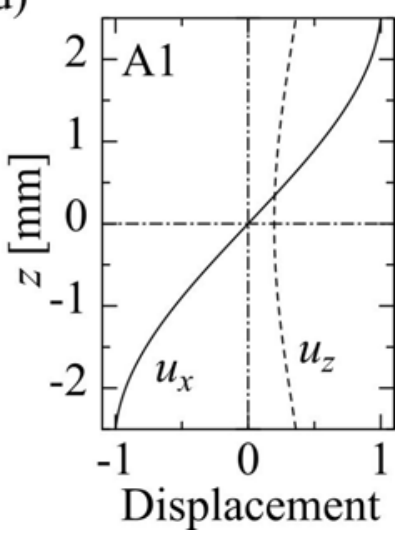

Fig. 15 Through-thickness displacement profiles for the (a) S0, (b) A0, (c) S1 and (d) A1 modes at 0.25 $\mathrm{MHz}$. 

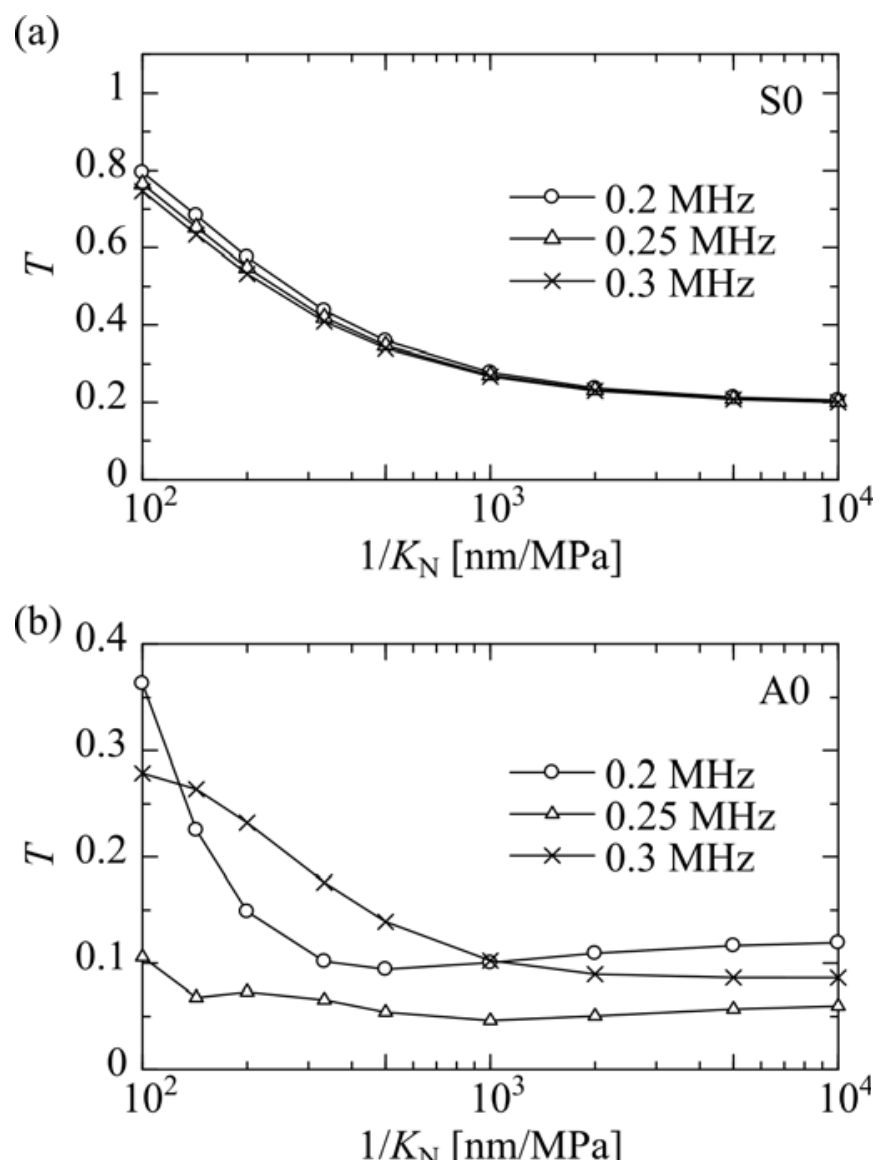

Fig. 16 Variation of the calculated transmission ratios of the (a) S0 and (b) A0 modes with the inverse of the interfacial stiffness $1 / K_{\mathrm{N}}$ at different frequencies. 